\title{
Spatial distortions within the Poggendorff figure and its variants: A parametric analysis
}

\author{
KATHRYN QUINA-HOLLAND \\ University of Wisconsin-Milwaukee, Milwaukee, Wisconsin 53201
}

\begin{abstract}
Angle size and horizontal separation of the Poggendorff figure and three components (acute angles, obtuse angles, and obliques) were varied parametrically. Two adult trained observers judged the distance between the obliques of the test figure relative to a pair of comparison dots during 250-msec tachistoscopic presentations. Results indicated: (1) the Poggendorff effect is created by an underestimation of intercontour distance, resulting in apparent misalignment; (2) only the obtuse angles can serve as a significant predictor of the classical illusion; (3) the parallel lines play an important contributory role in the Poggendorff illusion when they are present. Findings tended to support a theoretical explanation based on contour interactions.
\end{abstract}

The Poggendorff illusion is typically described as an illusion of misalignment: the upper oblique arm of a vertical figure appears to be placed "too high" relative to the lower arm, or the two diagonals do not appear to line up. In keeping with that description, studies of the Poggendorff have usually been concerned with the alignment of the two obliques along a single spatial coordinate. Most methods used to determine the magnitude of the illusion have required judgments about or adjustments to that alignment, and the extent of linear displacement along the parallel of the figure has been measured.

While this technique is descriptively sound, it overlooks the possibility that the misalignment may be due to some other form of contour displacement, and contains an implicit assumption that when alignment is correct, no figural distortion is active. To explore that possibility, we developed a technique which focused the observer's attention on intercontour distance, asking for judgments about the space between the obliques of the Poggendorff figure. By comparing the apparent intercontour distance for the test figure to that between a pair of comparison dots, a clearer picture of both the direction and extent of intercontour distortion was made possible.

In addition to giving a more precise record of the nature of the Poggendorff illusion, use of this technique offered a new look at older theories. For example, Gillam's (1971) application of perspective theory to the Poggendorff illusion predicts correct alignment of two oblique segments when no parallels

Based on a doctoral dissertation submitted to the University of Georgia, August 1973. Portions of this paper were presented at the 47th Annual Meetings, Midwestern Psychological Association, Chicago, Illinois, May 1-3, 1975. The author would like to thank Herschel Leibowitz, Robert H. Pollack, and Richard M. Warren for their helpful comments on this manuscript. are present. However, her theory suggests a distortion of the distance between those two obliques relative to a set of equidistant dots, because of the added cueing of depth (Gillam, Note 1).

Previous attempts to define component/whole relationships within the Poggendorff figure have relied upon a comparison of magnitudes of illusion for different forms of the figure (e.g., Coren, 1970) or a correlational approach (Day, 1973a). We, instead, recorded multiple judgments for a single subject across several components, allowing the application of multiple regression analyses to parametrically acquired data. With this procedure, not only could we calculate the extent to which each component correlated with the classical figure, but also we could determine the factor or combination of factors providing the best predictor of the classical illusion.

The components were selected for analysis so as to be consistent with previous literature on the Poggendorff and related figures. They were the acute angles, the obtuse angles, the transversal segments, and the parallel lines. Each will be described in turn.

\section{Acute Angles}

Early researchers attributed the Poggendorff effect to an overestimation of the acute angles (e.g., Wundt, 1898). More recent data indicate that the acute angles, in isolation, actually create an illusion reversed in direction from the classical figure (Restle, 1969), although Day (1973b) has produced evidence that the method used to determine the illusion influences the direction of the acute angles effect. Yet theories continue to appear which place special emphasis on the acute angles (e.g., Walker, 1973), and the work of Pressey and Sweeney (1972) suggests that the acute angle might even play a role in reducing the distortion in certain variants. 


\section{Obtuse Angles}

Eliminating part of the Poggendorff figure so that only the lines forming the obtuse angles remain results in an illusion whose magnitude and direction approach those of the classical figure (Day, 1973b; Pressey \& den Heyer, 1968). However, these studies have employed alignment in the dependent variable, and it remains to be seen whether the same relationship holds for the distance variable.

\section{Transversals}

Although the angles may play an important role in the Poggendorff, a number of observers have described a reduced Poggendorff-like displacement of the two transversals when neither parallel was present. Day (1973a) varied the angle of two transversal segments from vertical, and observed significant misalignment at $45^{\circ}, 60^{\circ}$, and $75^{\circ}$. Pressey and Sweeney (1969) demonstrated that slanting a single transversal towards a vertical line produced a misalignment about half as strong as, and highly correlated with, the classical Poggendorff illusion.

\section{Parallel Lines}

The findings of Quina and Pollack (1973) suggested that another variable might be at least influential in the Poggendorff illusion. In that study, an "attraction" effect was reported between a pair of parallel lines relative to a pair of comparison dots. The same form of displacement could operate within the classical Poggendorff illusion when parallel lines are present. While Weintraub and Tong (1974) have argued against the significance of this variable, they did find that observers consistently underestimated the space between the parallels for their figures. In addition to identifying a potential contributor to the illusion, the inclusion of the parallels allow some theoretical considerations about contour interactions not otherwise possible.

\section{METHOD}

\section{Subjects}

Two trained observers were paid for participation in the experiment. Both were male graduate students in their midtwenties. Each had 20/17 visual acuity, as measured by a Master Ortho-Rater, and no tested visual defects. Both had participated in an earlier and related experiment (Quina \& Pollack, 1973), so findings from that experiment could be utilized for the present study. Testing took place in daily sessions lasting 50 to $60 \mathrm{~min}$, including a break, over a period of 10 weeks.

\section{Apparatus and Stimuli}

For each of the component angular figures (see Figure 1), six angle sizes $\left(15^{\circ}, 30^{\circ}, 45^{\circ}, 60^{\circ}, 75^{\circ}\right.$, and $\left.90^{\circ}\right)$ were combined with two horizontal separations ( 5 and $11 \mathrm{~mm}$, respectively). The horizontal separations were selected from Quina and Pollack (1973) as two loci giving different degrees of attraction for two parallel lines. A total of 34 combinations of angle size and separation were involved $\left(90^{\circ}\right.$ angle nonreplicated for obtuse/ acute components). The classical figure was manipulated in similar fashion with angle size varied from $30^{\circ}$ to $75^{\circ}$.

Stimuli were drawn in black India ink, with a central gray fixation dot, on white $12.7 \times 17.8 \mathrm{~cm}$ cards. The test figure was drawn on one side of the dot, with black comparison points corresponding to the inner ends of the oblique lines equidistant to the other side. Lines were $0.5 \mathrm{~mm}$ wide and dots were approximately $1 \mathrm{~mm}$ in diameter. No figures exceeded a maximum of $2.5^{\circ}$ visual angle in the vertical direction and $3^{\circ}$ in the horizontal direction.

For each condition, a series of stimuli was constructed, varying the position of the upper comparison dot in .5-mm steps about that of the analogous test line. Control stimuli were exactly the same except that dots were substituted for the inner ends of the critical test lines.

Stimulus cards were shown in a three-channel tachistoscope

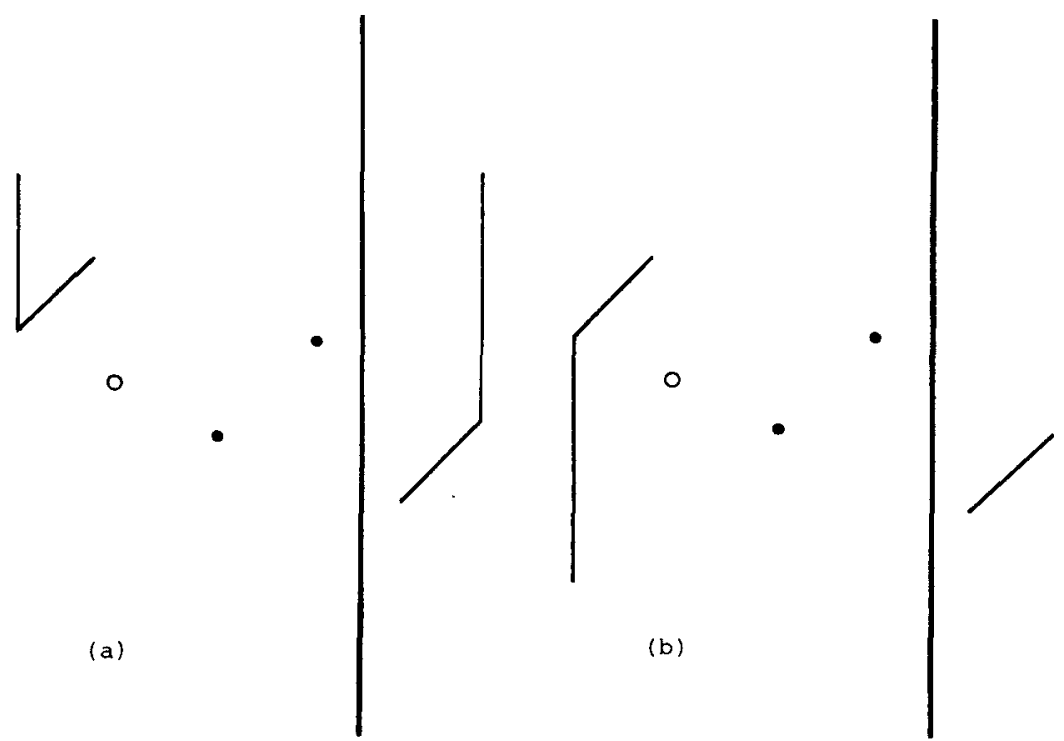

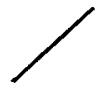

0

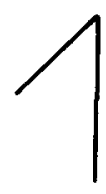

(a)

Figure 1. The stimulus figures used in the present study: (a) acute angles, (b) obtuse angles, (c) transversals. 
(Scientific Prototype, Model GB). Illumination was constant at 11.6 apparent foot candles, as measured by a Macbeth illuminometer.

\section{Procedure}

Participants began each session by adapting to the illumination level of the tachistoscope for $3 \mathrm{~min}$. A blank field containing only a center fixation dot was used during this period, and this field remained on throughout the session except during the stimulus presentations.

Stimuli were presented for $250 \mathrm{msec}$ in one field of the tachistoscope at an approximate rate of one every $4 \mathrm{sec}$. Stimulus series followed the method of constant stimulus differences, except that presentation was altered to allow trial-by-trial calculation of illusion magnitudes. A different random order was generated for the stimuli within each condition, control or experimental. Each presentation of a set of stimuli in this random order constituted a trial. For each condition, there were four control, eight experimental, and four final control trials.

Each series was shown to the observer under two orientation conditions, once with test lines to the left and once to the right of the center dot. Conditions and orientations within conditions were completely randomized, with the exception that the classical figure was not presented until after all other conditions were completed.

Observers were required to judge whether the amount of space between the comparison dots was greater or less than that between the test lines (or control dots). No "equal" judgments were allowed. No feedback was given as to the correctness of a judgment.

The responses were scored as follows: starting from the wider or narrower end of the series in the order $w, n, n, w$, across trials, that point located halfway between the last response consistent with the starting end and the first different response maintained for three consecutive settings was taken as the point of subjective equality (PSE). The mean control PSE across eight trials was subtracted from the experimental PSE to find the magnitude of illusion.

\section{RESULTS}

Illusion magnitudes were converted into percents by dividing by the actual distance between the inner ends of the obliques for each condition. Mean percents across eight trials for each component are presented in Figures 2 and 3 for the 11 - and $5-\mathrm{mm}$ conditions, respectively. For purposes of description, positive numbers indicate overestimation and negative numbers underestimation of the intertransversal space relative to the comparison dots.

As a rule, larger angular separations resulted in more stable trends, in the sense that functions were smoother and more regular. Orientation differences (right vs left) were infrequent within conditions; for the angular components, an average of 3.5 of 13 conditions per angle type and separation were found to be significantly different using $t$ tests $(p<.01)$, and most of these differences were in magnitude and not direction of trend. Differences in direction were significant in only 5 of the 78 comparisons.

For each angle type, data were analyzed in a threefactor analysis of variance for Angle Size by Horizontal Separation by Orientation, with follow-up trend tests. In each case, main effects of angle size and horizontal separation were significant at $p<.01$, but the presence of significant higher order interactions indicates use of the results of individual trend tests for statistical description of the angle size functions. All trend components reported were significant at the .01 level.

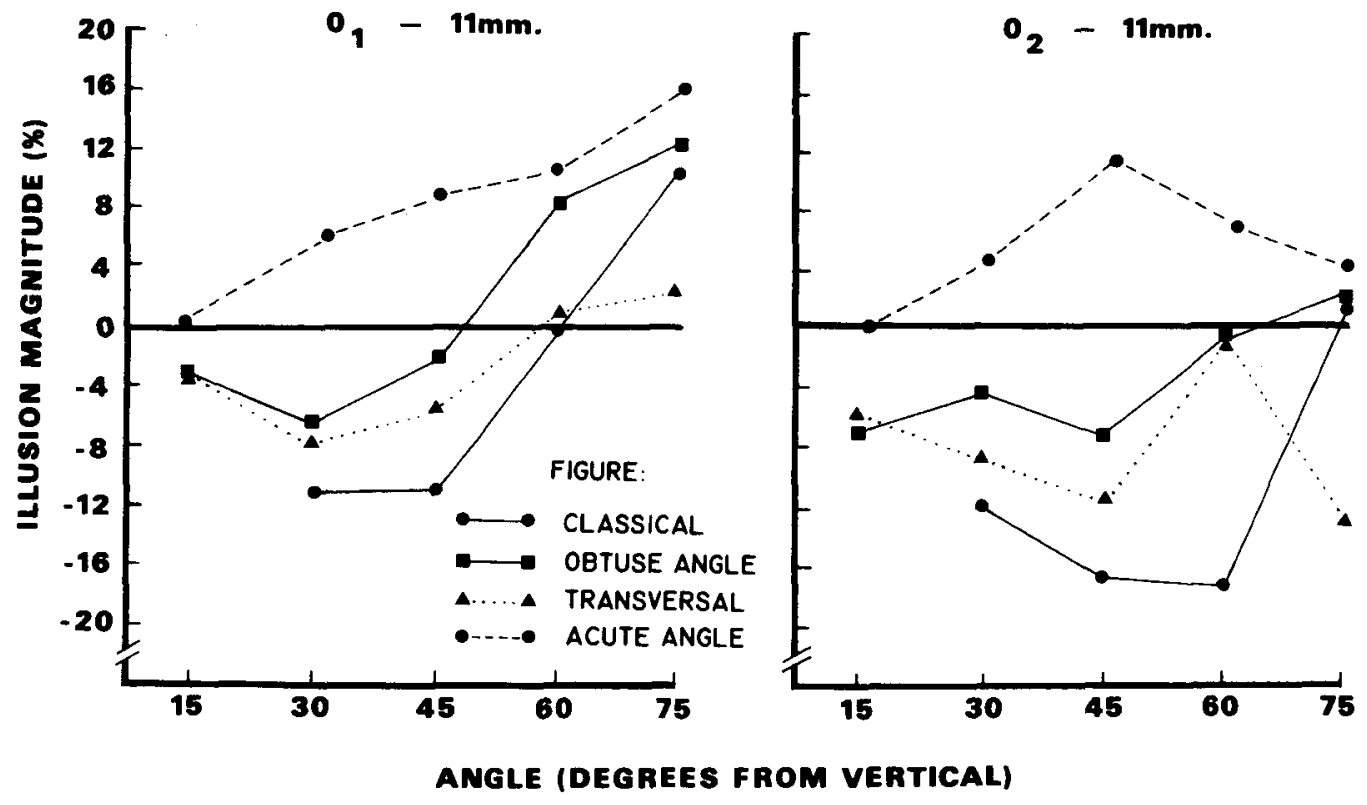

Figure 2. Mean percent illusion as a function of the angle formed by the oblique and the vertical lines, 11-mm separations. Positive numbers indicate overestimation of intercontour distance; negative numbers indicate underestimation. 

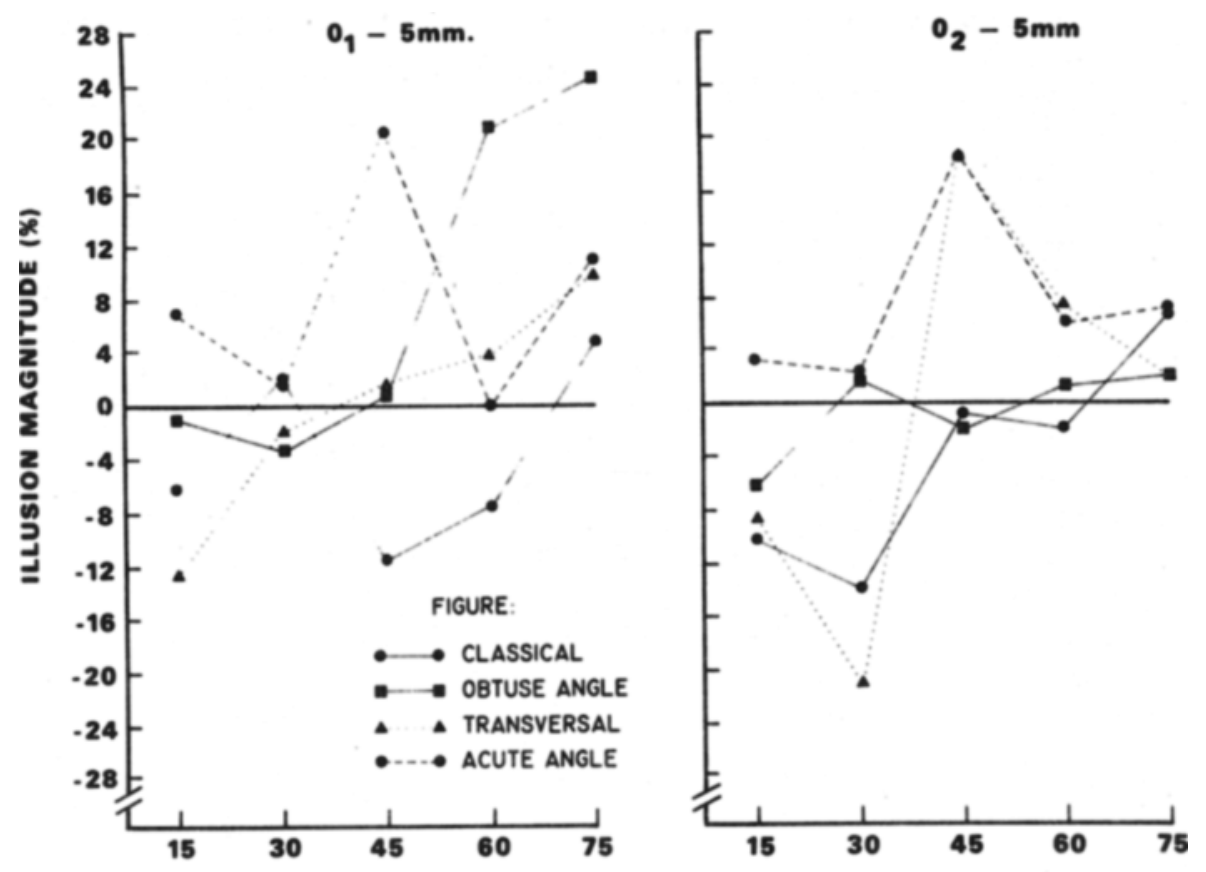

ANGLE (DEGREES FROM VERTICAL)

Figure 3. Mean percent illusion as a function of the angle formed by the oblique and the vertical lines, 5-mm separations. Positive numbers indicate overestimation of intercontour distance; negative numbers indicate underestimation.

\section{Acute Angles}

Most conditions produced an overestimation of the distance between the angles, with the misalignment described in the direction reported by Restle (1969) and others. For 11-mm separations, cubic trends predominated, with the percent of overestimation increasing between $15^{\circ}$ and $45^{\circ}-60^{\circ}$ conditions and declining to near zero at $90^{\circ}$. Trends were more complex for $5-\mathrm{mm}$ conditions, although intra- and interobserver consistency were evident. Maximum displacement was found for $45^{\circ}$ angles, with a drop to near zero at $60^{\circ}$. Overestimates of $10 \%-20 \%$ were recorded for both observers at $90^{\circ}$.

\section{Obtuse Angles}

Because of the need to standardize angles for comparison, the obtuse angles are presented in the figures in terms of their complementary acute angles. For both observers, the displacements for larger angles (over $135^{\circ}$ ) were negative; that is, the distance between the vertices was underestimated, and observers reported the misalignment in the normal direction. For smaller $\left(105^{\circ}-120^{\circ}\right)$ angles, the intercontour distance was overestimated, although this overestimation was slight for one observer. For observer 2, all trends were linear, with no significant higher order components. For observer 1, cubic components were also significant, largely reflecting the reduced displacements at $90^{\circ}$.

\section{Transversals}

When the obliques were tilted between $15^{\circ}$ and $45^{\circ}$ from the vertical, an underestimation of intercontour distance was found, with direction of misalignment corresponding to that reported for the classical figure. For 5-mm figures, the $60^{\circ}$ and $75^{\circ}$ angles produced an overestimation of the distance between the obliques, approaching zero at $90^{\circ}$. At $11 \mathrm{~mm}$, the distortions were near zero for larger angles, with the exception of a large underestimation at $75^{\circ}$ for observer 2 (that particular condition was highly reliable over three recordings).

\section{Classical Figure}

For 11-mm figures, the distance between the inner ends of the obliques was underestimated for angles up to $75^{\circ}$, where displacements were near zero or reversed. For the smaller horizontal separation, percents of illusion were smaller but the major effect was the same. Quadratic trends predominated in 11-mm conditions, and cubic trends in $5-\mathrm{mm}$ conditions.

\section{Angular Components}

To describe the relationships between the angular components and the classical figure, multiple regression analyses were performed on the data for each observer. ${ }^{1}$ The predictor variables were the acute angles, obtuse angles, and transversals; the 
criterion (dependent) variable was the classical figure. Each predictor variable was tested alone, then all possible combinations of the three components were entered and tested for predictive strength. These higher order tests produced partial $F$ values, with effects of other variables removed from the value for a single component. Partial $F$ values, when all variables were entered, indicated that only the obtuse angle component was a significant predictor [for observer $1, F(1,140)=8.21, \mathrm{p}<.005$; for observer $2, F(1,140)=17.39, p<.0001]$. While the transversal component was highly correlated with the obtuse angle for observer $1(\mathrm{r}=.570$ with $\mathrm{df}=142$, $p<.01$ ), the partial $F$ for the transversal predictor was not significant $(p>.35)$. The obtuse angle component accounted for $32.79 \%$ and $34.23 \%$ of the variance in the classical figure for observer 1 and observer 2 , respectively.

\section{Parallel Lines}

The effect of adding parallel lines to the Poggendorff figure was assessed by comparing percent of illusion from the obtuse-angle figure to those from the classical figure. In addition, figures separated by $5 \mathrm{~mm}$ were compared to those separated by $11 \mathrm{~mm}$, since earlier work had shown that attraction effects were greater for the latter. A 2 by 2 ANOVA for type of figure and horizontal separation was performed for each observer. Results for observer 2 supported both hypotheses. The classical illusion figure consistently produced greater underestimation (or less overestimation) than the obtuse angle figure $[F(1,28)=5.73, p<.025]$, and the 11 -mm figure produced greater underestimation (less overestimation) than the 5-mm figure $[F(1,28)=5.71$, $\mathrm{p}<.025$ ]. The interaction was not significant. For observer 1, the classical illusion was more negative than the obtuse angle figure $[F(1,28)=7.14$, $\mathrm{p}<.025$, but no difference was found for 5-vs $11-\mathrm{mm}$ figures. ${ }^{2}$ Again, the interaction was not significant.

\section{DISCUSSION}

The significance of these data lie in three findings. First, the distortion obtained for the Poggendorff figure was, for most conditions, an underestimation of the distance between the obliques, relative to that between the comparison dots. This demonstrates that the illusion is not a line appearing to be placed "too high," but that, in fact, the apparent misalignment is produced by a factor causing the elements of the figure to appear closer together.

In addition, distortion of the apparent distance was found when alignment was correct, for $90^{\circ}$ angles. These data reveal serious shortcomings of previous methods of describing the illusion, and indicate that the distance factor should be con- sidered in future evaluations of the Poggendorff illusion.

Secondly, the multiple regression analyses clearly indicated that only the obtuse angles provide ample predictive power for the classical illusion. While the parallelless variant underwent a distortion similar in direction to that of the classical figure, its lack of predictive utility indicates that it represents a separate type of effect.

Finally, the parallel factor in the present study yielded important information. The classical figure, containing completed parallel lines, produced greater underestimation (and less overestimation) of the intercontour distance than the obtuse angles figure. This suggests that the parallels play some role in determining the total magnitude of illusion in the classical figure.

\section{Theoretical Considerations}

The present findings afford no support for theories of the Poggendorff which rely upon general spatial factors, such as assimilation of oblique lines to the nearest cardinal axis (e.g., Goldstein \& Weintraub, 1972), or incorrect projection across the diagonal space (Krantz \& Weintraub, 1973). Rather, they limit appropriate theoretical explanations to those encompassing obtuse and complete figures within the same descriptive concept, such as those of Gillam (1971), Pollack (1964), and Pressey (1971).

Pressey (1971) has developed an assimilation theory of optical illusions which relies upon the attentional context within which the distortion takes place. According to assimilation theory, the observer projects a series of possible magnitudes in any judgment of length or size. Judgments are then based on the average of the components in the projected series. The Poggendorff illusion, in its normal symmetrical form, creates a context in which magnitudes are projected between the obliques and the opposite parallel. Because of the central attentional focus, the projected connecting lines are shorter than the actual connecting line, resulting in an underestimation of the true path. Thus the space between the inner ends of the transversal is effectively shortened, and the extension of each oblique appears to lie closer than the objectively continued line.

Assimilation theory predicts the present findings of maximal similarity between obtuse angles and the classical figure, and also the underestimation of the intercontour space. One conflict which may be of theoretical significance, however, is the fact that illusion magnitudes observed for the classical figure were greater (more negative) than those for the obtuse angles. While Pressey has not addressed this issue directly, his descriptions (e.g., Pressey \& Sweeney, 1972) suggest that an extension of the parallels beyond the point of intersection with the 
obliques, as in the classical figure, would draw additional projected magnitudes which would be longer than the true magnitudes. Thus the amount of shrinkage should be slightly less in the classical figure than in the obtuse-angle figure, where all projected magnitudes would be shorter than the true distance. Nevertheless, the influence of attentional context on perceptual judgments is a valuable concept which deserves further attention.

The finding that the obtuse angle was the most significant predictor also matches requirements of Gillam's (1971) three-dimensional depth-processing theory. Gillam has applied Gregory's (1963) "misapplication of size constancy" framework to the Poggendorff illusion. According to her theory, the presence of overlapping parallel lines causes the two obliques to be incorrectly processed as lying horizontally and projecting forward or backward into space. Since the lines appear to lie in different horizontal planes, they cannot be collinear, and thus they are viewed as two unrelated segments. Apparent noncollinearity can be translated into misjudgments of apparent distance if one assumes that the comparison dots in nonhorizontal orientations are likely to give rise to the impression of depth, and thus increased apparent distance between them. The dots would appear further apart than two points lying on the same horizontal plane, as would be represented in the classical figure.

The theory and formulae proposed by Gillam assume that two lines overlapping enough to be perceived as coplanar are necessary for the classical form of the Poggendorff illusion. Only the larger obtuse angles fulfill that overlap, in keeping with the present results. However, two findings in the present study are not consistent with a depth processing theory. One is the presence of distortions for $90^{\circ}$ angles in the smaller figures, where both lines and dots should appear coplanar and hence accurate. The other is the overestimation of distance found for the classical figure when angles approached the horizontal. The depth processing theory seems restricted to an underestimation effect for the classical figure across all angle sizes under $90^{\circ}$.

Pollack (1964) suggested a dual-process view of contour interactions which would place emphasis on parallel contours as well as the angles in identifying the components of the Poggendorff illusion. Concerned with distortions of the space between neighboring contours, Pollack predicted that parallel contours are displaced toward each other (attraction), while contours whose projections intersect would be displaced away from each other (repulsion). These functions should follow the distance paradox of increasing, then decreasing magnitude with increasing separation of contours, and are assumed to have an underlying neurophysiological origin.

Quina and Pollack (1973) confirmed the existence of an attraction effect for parallel contours, finding that the space between a pair of vertical lines was underestimated relative to the space between a pair of equidistant comparison dots. That underestimation effect followed the distance paradox, increasing in magnitude up to a contour separation of about $1 / 2^{\circ}$ visual angle (11 mm, as in the present study) and declining ther eafter.

The present results could be interpreted according to simultaneous attraction of parallel and repulsion of perpendicular contours. The effect obtained with most of the conditions for larger obtuse angle and classical figures was an underestimation of the intervening space. In those figures, the major portion of the overlapping contours were parallel to each other. For angles close to $90^{\circ}$, an overestimation of the space was observed, suggesting that projected intersection of these contours produced a strong (and dominant) repulsion effect. The acute angles were likewise displaced away from each other, following the predictions for perpendicular contours.

The results of the comparison of illusion magnitudes for classical and obtuse angle figures may be taken as strong evidence for Pollack's hypotheses, since increasing the extent of overlapping parallel contours increased the illusion effect. The larger illusions observed for the 11-mm separation for observer 1 lends additional, albeit weak support, as that is consistent with the data from Quina and Pollack (1973).

In conclusion, the value of the application of multiple regression techniques to a parametric design has been demonstrated by a more discriminating view obtained of the component/classical relationships than any provided by previous research. Although distortion for the obliques superficially resembles that for the classical figure, the illusion does not appear to rely upon mechanisms which apply to isolated features. When the entire parametric function is considered, only the obtuse angles figure stands as a valid predictor of the classical illusion. The explanatory concept, therefore, must be one which involves a more complete figure and a set of distortions appropriate for the complex angular configurations.

But while the obtuse angle components clearly dominated the illusion in the present analyses, considerable variability was left unaccounted for. One step towards the accountability involves the definition of previously overlooked contributory aspects, such as the parallel lines. While not responsible for the illusion, the results indicate that the parallels play an important role in the classical figure by providing a juxtaposition of contours which acts to increase the magnitude of the underestimation effect (or decrease the overestimation effect).

Finally, the development of a technique which 
relies upon distance judgments rather than alignment reveals an effect of theoretical significance in the explanation of this and other illusions: the distortion of distance between elements of a figure existing independently from their alignment. Taken together, the results point to the necessity for explanations of illusions based on interactions of elements within the total configuration. What is needed is a set of rules which are general enough to encompass distortions in variants and components of the larger figure, including reversals as in the acute angles figure, but at the same time specific enough to describe the peculiar configuration of the obtuse angles. At the present time, the attraction-repulsion contour interaction theory seems to fit these requirements most adequately.

\section{REFERENCE NOTE}

1. Gillam, B. Personal communication, April 25, 1976.

\section{REFERENCES}

Coren, S. Lateral inhibition and geometric illusions. Quarterly Journal of Experimental Psychology, 1970, 22, 274-278.

DAY, R. H. The oblique line illusion: The Poggendorff effect without parallels. Quarterly Journal of Experimental Psychology, 1973, 25, 535-541. (a)

DAY, R. H. The Poggendorff illusion with obtuse and acute angles. Perception \& Psychophysics, 1973, 14, 590-596. (b)

Grllam, B. A depth processing theory of the Poggendorff illusion. Perception \& Psychophysics, 1971, 10, 211-215.

Goldstein, M. B., \& Weintraub, D. J. The parallel-less Poggendorff: Virtual contours put the illusion down but not out. Perception \& Psychophysics, 1972, 11, 353-355.

GREGORY, R. L. Distortion of visual space as inappropriate constancy scaling. Nature, 1963, 199, 678-680.

Krantz, D. H., \& Weintraub, D. J. Factors affecting perceived orientation of the Poggendorff transversal. Perception \& Psychophysics, 1973, 14, 511-517.
Pollack, R. H. The effects of fixation on the apparent magnitude of bounded horizontal extents. American Journal of Psychology, 1964, 77, 177-192.

Pressey, A. W. An extension of assimilation theory to illusions of size, area, and direction. Perception \& Psychophysics, 1971, 9, 172-176.

Pressey, A. W., \& den Heyer, K. Observations on Chiang's "new" theory of geometrical illusions. Perception \& Psychophysics, 1968, 4, 313-314.

Pressey, A. W., \& Sweeney, O. A variation of the Poggendorff illusion. Perceptual and Motor Skills, 1969, 28, 883-886.

Pressey, A. W., \& Sweeney, O. Some puzzling results on the Poggendorff illusion. Perception \& Psychophysics, 1972, 12, 433-437.

Quina, K., \& Pollack, R. H. Attraction of parallel lines as a function of intercontour distance. Perceptual and Motor Skills, 1973, 36, 934.

Restle, F. Illusions of bent line. Perception \& Psychophysics, 1969, 5, 273-274.

WALKER, E. H. A mathematical theory of optical illusions and figural aftereffects. Perception \& Psychophysics, 1973, 13, 467-486.

Weintraub, D. J., \& Tong, L. Assessing Poggendorff effects via collinearity, perpendicularity, parallelism, and Oppel (distance) experiments. Perception \& Psychophysics, 1974, 16, 213-221.

Wund, W. Die geometrisch-optischen Taeuschungen. Abhandlungen der mathematisch-physischen Classe der koeniglischen Saechischen Gesellschaft der Wissenschafien, 1898, 42, No. 2 (translated by D. J. Carter).

\section{NOTES}

1. The author extends her appreciation to the University of Wisconsin-Milwaukee Social Science Research Facility for assistance in the analyses.

2. In reviewing the data for observer 1 from Quina and Pollack (1973), it was found that he had obtained the smallest difference between the 5 - and $11-\mathrm{mm}$ conditions of the participants in that study.

(Received for publication December 1, 1975; revision received November $1,1976$. 